\title{
Very early onset schizophrenia in Dandy-Walker variant: A case report
}

D B Hettiarachchi, E Gunarathne, C Hettiarachchi

\section{Abstract}

The Dandy-Walker variant is a milder form of the Dandy-Walker Complex (DWC) with less severe radiological abnormalities. We describe a 13-year old girl with limited intellectual capacity who presented with clinical features of schizophrenia, and who was diagnosed with the Dandy-Walker variant during organic screening for first episode psychosis.

Key words: schizophrenia, early onset, Dandy-Walker variant

SL J Psychiatry 2021; 12(2): 36-37

\section{Introduction}

The Dandy-Walker complex (DWC) is a series of neurodevelopmental anomalies in the posterior fossa, including the Dandy-Walker malformation, DandyWalker variant (cerebellar hypoplasia and cystic dilatation of the fourth ventricle), mega-cisterna magna and posterior fossa arachnoid cyst (1). The Dandy-Walker variant is a milder form of DWC with less severe radiological abnormalities, including the absence of hydrocephalus, less neurological symptoms, and a better outcome (1). There are only a few case reports on the cooccurrence of schizophrenia like psychosis and DWC (2). Dandy-Walker variant may also be associated with mood symptoms, obsessive compulsive symptoms, hyperactivity and impulsive behavior (3).

We describe a 13-year old girl with limited intellectual capacity who presented with clinical features of schizophrenia, and who was diagnosed with the Dandy-Walker variant during organic screening for first episode psychosis.

\section{Case Report}

A 13-years old girl was brought to the child and youth mental health services due to suspiciousness, muttering to self and running away from home for a period of 3 months, that had gradually increased in severity. The mother claimed that the child's birth and development had been normal. The child did not have any significant medical comorbidity. There was no family history of mental health issues.

On mental state examination she was found to be experiencing systematized persecutory delusions, where she strongly believed that a group of teachers at her school were trying to poison the food she eats at home. She was experiencing third person auditory hallucinations, where she used to hear this group discussing about her at school, which was more than 10 kilometers away from her home. She also believed that they had fixed a camera at home to monitor her behaviors.

According to Tests of Nonverbal Intelligence-4 (TONI 4) she presented with poor intelligence level [Index score -73, Normal range 90-110]. Her general examination and neurological examination were normal. Her hematological and biochemical investigations including the thyroid profile were normal, and the presence of Kayser-Fleischer rings were excluded. Electroencephalogram (EEG) suggested mild cerebral dysfunction. Computed tomography (CT) scan of the brain reported a retro cerebellar cerebrospinal fluid (CSF) density lesion communicating with the fourth ventricle. However, CT report also noted a normal posterior fossa, suggesting the Dandy-Walker variant.

She was diagnosed to have schizophrenia and commenced on risperidone $1 \mathrm{mg}$ at night and managed as an outpatient. Since her symptoms did not improve after 2 weeks, the risperidone was increased to $2 \mathrm{mg}$ daily. Only a slight improvement was seen with risperidone $2 \mathrm{mg}$ after three weeks, and therefore the dose was gradually increased up to $2 \mathrm{mg}$ two times per day. Since her psychotic symptoms showed only a partial response and she continued to have odd behaviors with poor functional level and school refusal after 3 months of treatment, the antipsychotic was changed to olanzapine $5 \mathrm{mg}$ two times per day, with the plan to further increase the dose depending on response. No active neurosurgical interventions were indicated at this stage. 


\section{Discussion}

The relationship between psychotic disorders, in particular, schizophrenia, and neurodevelopmental abnormalities has been reported in recent literature (2). However, the relationship between DWC and psychosis has not been well studied, possibly because of the rarity of this condition. Even though many isolated case reports of psychosis and DWC, with regards to adult patients, are reported in literature, there are only a few case reports related to adolescents (4). Psychosis associated with DWC is described to be characterized by juvenile or young adult onset, atypical psychotic symptoms, high frequency of family history of psychosis, high prevalence of cognitive deficit, and refractoriness to treatment according to case studies (4). From amongst these features, the child we describe here had early onset psychotic symptoms, low intellectual capacity and partial response to treatment.

As in the case of our patient, schizophrenia and DandyWalker variant may be found coincidentally together, or any cerebellar dysfunction due to Dandy-Walker variant may cause or contribute to the appearance of psychotic symptoms. The relationship between schizophrenia and cerebellar malformations has been documented in previous research studies (5). Abnormalities of the cerebellum, especially in the vermis, have been linked with the pathophysiology of schizophrenia and have been detected not only in patients with a long-standing illness, but also at the time of onset of psychotic symptoms (6).

\section{Conclusions}

This clinical case emphasizes the need for a complete evaluation, including neuroimaging studies, in children or adolescents presenting with first-episode psychosis, even if the clinical picture is strongly suggestive of a clear psychiatric condition. It also highlights the importance of being aware of psychiatric manifestations of cerebellar disease, as it may have impact on patient management as well as the prognosis. Further research is required to establish the best pharmacological options for patients with similar neurological conditions who do not require neurosurgical interventions.

The patient's mother gave informed consent for the publication of the findings in this case report.

\section{Acknowledgments}

The authors acknowledge the patient and her family, for their support for the preparation of this case report.

\section{Conflicts of interest}

There are no conflicts of interest.

\section{Author contributions}

$\mathrm{DH}$ wrote the initial draft, $\mathrm{AG}$ and $\mathrm{CH}$ contributed to the management of the patient, and editing of the manuscript. All authors have seen and approved the final manuscript.

D B Hettiarachchi, E Gunarathne, C Hettiarachchi, Child and Adolescent Psychiatry Unit, Teaching Hospital, Karapitiya

Corresponding author: D B Hettiarachchi

Email: Darshani.hettiarachchi@gmail.com

http://orcid.org/0000-0001-5381-6116

\section{References}

1. Barkovich AJ, Kjos BO, Norman D, Edwards MS. Revised classification of posterior fossa cysts and cystlike malformations based on the results of multiplanar MR imaging. Am J Roentgenology 1989; 153: 1289-300.

2. Turner SJ, Poole R, Nicholson MR, Ghadiali EJ. Schizophrenia-like psychosis and Dandy-Walker variant. Schizophr Res 2001; 48: 365-7.

3. Prakash R, Kour J, Singh S A. Psychiatric Comorbidities in Dandy-Walker Variant Disorder. The Journal of Neuropsychiatry and Clinical Neurosciences. October 2009. DOI: 10.1176/appi.neuropsych.21.4.477.

4. Gan Z, Diao F, Han Z, Li K, Zheng L, Guan N, et al. Psychosis and Dandy- Walker complex: report of four cases. Gen Hosp Psychiatry 2012; 34: 102.e7-102.e11.

5. Joyal CC, Pennanen C, Tiihonen E, et al. MRI volumetry of the vermis and the cerebellar hemispheres in men with schizophrenia. Psychiatry Res 2004; 131: 115-24.

6. DeLisi LE, Sakuma M, Tew W, et al. Schizophrenia as a chronic active brain process: a study of progressive brain structural change subsequent to the onset of schizophrenia. Psychiatry Res 1997; 74: 129-40. 